\title{
Características de las PyMEs según la gerencia sea hombre o mujer: Caso empírico de Cali
}

\author{
Mónica García Solarte ${ }^{1}$ \\ Universidad del Valle \\ monica.garcia@correounivalle.edu.co \\ Carlos Hernán González Campo² \\ Universidad del Valle \\ carlosh.gonzalez@correounivalle.edu.co \\ Guillermo Murillo Vargas ${ }^{3}$ \\ Universidad del Valle \\ guillermo.murillo@correounivalle.edu.co
}

DOI: https://doi.org/10.21158/01208160.n82.2017.1648

Cómo citar este artículo/ To reference this article/ Comment citer cet article/ Para citar este artigo:

García, M.; González, C. H. y Murillo, G. (2017). Características de las pymes según la gerencia sea hombre o mujer: caso empírico de Cali. Revista EAN, 82, pp.143-160. https://doi.org/10.21158/01208160.n82.2017.1648

\section{Resumen}

El objetivo de esta investigación es caracterizar el comportamiento de las PyMEs según la gerencia sea hombre o mujer. Para lograr el objetivo, se utilizó una metodología de enfoque cuantitativo, en la que se aplicó una encuesta estructurada a 142 gerentes de Cali. Los resultados obtenidos dan cuenta de que las PyMEs que son dirigidas por mujeres son pocas, y presentan, entre otras, las siguientes características: son de mayor tamaño; tienen menor participación femenina dentro de sus empleados, pero mayor participación dentro de las juntas directivas; no pertenecen ampliamente a organizaciones de carácter familiar; tienen mayores socios o dueños hombres y su nivel de formación mayoritariamente es en estudios básicos. El aporte fundamental de esta investigación radica en la apertura de nuevos campos de conocimiento en Colombia donde el tema de gerencia no ha sido ampliamente investigado especialmente cuando las mujeres ocupan este tipo de cargos.

\section{Palabras clave}

Gerencia, PyMEs, carácter familiar, junta directiva, propiedad.

${ }^{1}$ Ingeniera industrial de la Universidad del Valle. Magíster en Administración de Empresas por la Universidad del Valle. Master of Management de Tulane University. Doctora en Dirección de Empresas por la Universidad Politécnica de Cartagena -España-. Profesora del Departamento de Administración y Organizaciones de la Facultad de Ciencias de la Administración de la Universidad del Valle.

2 Economista de la Universidad del Valle. Doctor en Administración de la Universidad EAFIT. Magíster en Ciencias de la Organización de la Universidad del Valle. Profesor del Departamento de Administración y Organizaciones de la Facultad de Ciencias de la Administración de la Universidad del Valle.

3 Administrador de Empresas de la Universidad del Valle. Doctor en Administración de la Universidad EAFIT, con el apoyo de HEC de Montreal-Canadá. Magíster en Ciencias de la Organización de la Universidad del Valle. Profesor del Departamento de Administración y Organizaciones de la Facultad de Ciencias de la Administración de la Universidad del Valle. 


\title{
Features of PyMES according to male or female management: An Empirical Case in Cali
}

\begin{abstract}
This research paper describes the behavior of PyMES according to male or female management. To reach its aim, a quantitative methodology was used and a well-structured survey was applied to 142 managers in Cali. Its outcomes reveal that there are few PyMES directed by women and they have the following features: they have a bigger size; they don 't have a major female participation in staff, but they have a major participation in the executive board; they have more male shareholders; and their educational level is basically primary and secondary studies. Its most important contribution reveals an aperture of new fields of knowledge in Colombia regarding management and leadership, which specifically haven 't been widely explored when women are part of the company executive boards.
\end{abstract}

Key words. Management, PyMES, family character, executive board, property.

\section{$\longrightarrow$ \\ Caractéristiques des PME selon que le dirigeant soit un homme ou une femme: cas empirique de la ville de Cali}

Resumé. L'objectif de cette recherche est de caractériser le comportement des PME selon que le dirigeant soit un homme ou une femme. Pour atteindre cet objectif, nous avons utilisé une méthodologie quantitative dans laquelle une enquête structurée a été soumise à 142 dirigeants d'entreprises de la ville de Cali. Les résultats obtenus rendent compte que les PME dirigées par des femmes sont peu nombreuses, et présentent, entre autres, les caractéristiques suivantes: elles sont de taille plus grande, la représentation féminine des employés est moindre, mais plus de femmes font partie des équipes de direction; ce ne sont généralement pas des organisations à caractère familial; les associés ou propriétaires sont de sexe masculin et leur niveau de formation correspond majoritairement à des études du secondaire. L'apport fondamental de cette recherche réside en la mise à jour de nouveaux champs de la connaissance dans lesquels le thème de la gestion n'a pas été amplement investigué lorsque des femmes occupent les postes de dirigeantes.

Mots clefs. Gérance, $P M E$, caractère familial, équipe de direction, propriété.

\section{Características das pequenas e médias empresas segundo a gerência de homem ou mulher: o caso empírico da cidade da cidade de Cali}

Resumo. NO objetivo desta pesquisa é a de caracterizar o comportamento das pequenas e médias empresas segundo a gerência de homem ou mulher. Para conseguir o objetivo, utilizou-se uma metodologia de enfoque quantitativo na qual se aplicou um questionário estruturado a 142 gerentes de Cali. Os resultados obtidos revelam que as pequenas e médias empresas dirigidas por mulheres são poucas, e apresentam, entre outras, as seguintes características: são de maior tamanho; têm menor participação feminina dentro de seus empregados, mas maior participação dentro das juntas diretivas; em geral não pertencem a organizações de caráter familiar; têm e sua maioria sócios ou donos homens e seu nível de formação em sua maioria é de estudos básicos. A contribuição fundamental desta pesquisa está na abertura de novos campos de conhecimento na Colômbia onde o tema de gerência não tem sido amplamente pesquisado especialmente quando as mulheres ocupam este tipo de cargos.

Palabras chave. Gerencia, PMEs, caráter familiar, junta diretiva, propriedade. 


\section{Introducción}

A lo largo del tiempo los roles de género han estado marcados por características muy propias. Los hombres han sido reconocidos por su poder, competencia y autoridad, sin embargo, las mujeres se han asociado a cuestiones sociales y humanas (Eagly, 1987). Actualmente, los asuntos de género dentro de la comunidad y las organizaciones han sido analizados y reevaluados. La participación de las mujeres en el mercado laboral ha aumentado y la reglamentación en países latinoamericanos ha tenido avances significativos. Muchas mujeres a lo largo del tiempo han empezado a tener cargos ocupados por los hombres y que anteriormente no eran de su acceso. En lo empresarial, las organizaciones dirigidas por mujeres también han aumentado, aunque no de manera tan significativa como se ha pretendido, ya que el rol de la mujer en su vida familiar, según la metáfora de "techo de cristal" (BarnetVerzar y Wolf, 2008), impide el acceso a cargos estratégicos y de dirección debido a su condición en la sociedad.

La gestión de la gerente mujer crea en la organización ciertas características distintivas a las gestionadas por los hombres. Las investigaciones en cuanto a la gerencia ser hombre o mujer son amplias, empero su aplicación en las PyMEs sigue siendo limitada, especialmente en Colombia. En tal sentido, el objetivo de esta investigación es caracterizar el comportamiento de las PyMEs de Santiago de Cali según la gerencia sea hombre o mujer, y para el caso pretende mostrar un análisis descriptivo de las variables seleccionadas en cuanto a la gestión según sea hombre o mujer.
Los resultados de esta investigación evidencian que las pymes gestionadas por mujeres tienen características diferentes de las gestionadas por los hombres. Los resultados muestran que las empresas que son dirigidas por mujeres son pocas en los sectores de manufactura y alta tecnología, las PyMEs son de mayor tamaño en cuanto a número de empleados, tienen menor participación femenina en la fuerza laboral, no suelen ser de carácter familiar, tienen mayores socios o dueños hombres y el nivel de formación de estas gerentes es mayoritariamente en estudios básicos. Estos resultados muestran que la participación de la mujer dentro de las organizaciones va en aumento con relación a los hombres, a pesar de que el acceso para estas a posiciones estratégicas aún actúa bajo el techo de cristal. Esta característica no posibilita que la mujer alcance logros profesionales tanto o más altos que los hombres. La presente investigación contribuye a los estudios de las mujeres y los hombres, y a la adopción de nuevas medidas de gestión y reglamentaciones enfocadas a la participación de la mujer en el mercado laboral.

En primer lugar, este artículo muestra la revisión teórica que se ha hecho respecto del género y los estudios a los que se ha asociado el sector, el tamaño y la edad de la organización, el carácter familiar, los socios, la junta directiva y el nivel de formación con relación a la gerencia de los hombres y las mujeres. En segundo lugar, se presenta la metodología seleccionada, el cálculo de la muestra y la descripción de las variables. En tercer lugar, se hace un análisis de los resultados de manera descriptiva para finalmente exponer las principales conclusiones generadas por el estudio. 


\section{Marco teórico}

- concepto de género ha sido trabajado de - diversas maneras a lo largo del tiempo. Lamas (1994), plantea el género como la simbolización cultural que se construye a partir de la diferencia sexual, dada desde el nacimiento, y que se hace evidente en la vida social, económica y política. En este sentido, el género es lo que significa ser hombre o mujer, y su relación con lo masculino y lo femenino; así mismo, cómo este hecho define las oportunidades, los papeles, las responsabilidades y las relaciones entre las

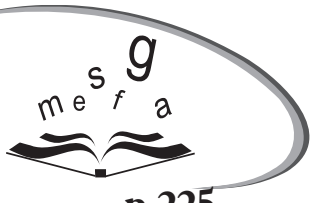

p. 225 personas, dentro de una comunidad u organización. Bajo la idea de ser hombre o mujer, los trabajos de Driga y Prior (2010) y Díaz y Jiménez (2010) identifican la gerencia de acuerdo con si son hombre o mujer con las características de las organizaciones: con estos autores se toma el concepto para la presente investigación.

Ahora bien, se ha reconocido a los hombres centrándolos en roles que resaltan el poder, la competencia y la autoridad. En contraste, las mujeres se han caracterizado por tener roles relacionados con apoyo social e interacciones humanas (Eagly, 1987). En un principio, Moser (1989), sostenía que las mujeres realizan tres funciones en la sociedad: reproductivo, productivo y gestión de la comunidad. Durante un tiempo estas funciones fueron totalmente aceptadas dadas la posición que ocupaban las mujeres y sus desigualdades económicas, políticas y de acceso al trabajo. Sin embargo, actualmente, la cuestión de género dentro de la comunidad y las organizaciones ha sido reevaluada y ha dado aportes de distinta índole. De acuerdo con la teoría de roles sociales de Eagly
(1987), las características diferenciales atribuidas a hombres y mujeres es desigual desde el punto de vista de las ocupaciones profesionales y familiares.

Alrededor del mundo, la participación de las mujeres en el mercado laboral se ha mantenido estable y se ha situado en $52 \%$. Por el contrario, las tasas de participación en la fuerza laboral, mundialmente para los hombres han disminuido de forma constante de $81 \%$ a $77 \%$. En este sentido, las brechas de género, y tal como lo menciona el Department of Economic and Social Affairs (2010), cada día son menores y con el tiempo las mujeres han empezado a ocupar posiciones masculinas. Según The Global Gender Gap Report 2015, la diferencia de participación de hombres y mujeres mundialmente para 2015 situó a Colombia en el puesto 35 entre 133 países analizados, lo cual indica que el país se encuentra dentro de un nivel en el que el hombre y la mujer tienen participación similar en diferentes ámbitos. Los indicadores más débiles de este informe son la participación de la fuerza laboral femenina, pese a que el número de matriculados de hombres y mujeres en educación universitaria y la ocupación de los legisladores, altos funcionarios y directivos ocupen el primer puesto.

Los indicadores anteriores muestran que Colombia ha tomado mayor fuerza y está más reglamentada en asuntos de género. $E L$ País creó la Consejería Presidencial para la Equidad de la Mujer, igualmente, en su Plan Nacional de Desarrollo, estableció su política Mujeres Constructoras de Paz y Desarrollo. Además, La Ley 731 de 2002 apoya las actividades de las mujeres en sectores como 
turismo rural y ecológico, artesanías, joyería, así como la comercialización, transformación de productos y prestación de servicios con relación a esos sectores. La Ley 823 de 2003 reglamenta el otorgamiento de apoyo tecnológico, organizacional y gerencial a las micro, PyMEs dirigidas por mujeres y a las que empleen en su mayoría personal femenino.

Estos indicadores han generado cambios significativos en la estructura de la fuerza laboral y su organización, sobre todo en la búsqueda del derecho de igualdad en oportunidades. Empero, en las diferentes esferas empresariales aún se evidencia la persistencia de la desigualdad entre hombres y mujeres (Watson, 2002; Cuadrado y Morales, 2007; Fernández, López, Maeztu y Martín, 2010; Castaño, Martín, Vázquez y Martínez, 2010; Fuentes y Sánchez, 2010; Torres y Pau, 2012). La concentración de las mujeres en determinados sectores y puestos de trabajo sigue estando muy marcada, en los que las mujeres ocupan, por lo general, empleos que son considerados "no estratégicos" (Schein, Mueller y Jacobson, 1989, Fernández et al., 2010).

En este sentido, la metáfora techo de cristal (Barnet-Verzar y Wolf, 2008) es una de las principales teorías a la que se le atribuye el estudio de la situación de desigualdad de oportunidades y discriminación. Esta metáfora es utilizada para designar una barrera invisible que impide a las mujeres cualificadas alcanzar puestos de mayor nivel dentro de las organizaciones y de responsabilidad.

Cuadrado y Morales (2007) y Castaño et al. (2010), muestran que ni la mayor participación de las mujeres en el mercado laboral, ni las medidas legales adoptadas, ni la formación académica recibida han generado un aumento proporcional de la representación femenina en puestos gerenciales, tal como aparentemente los indicadores muestran. Esta situación ha dado lugar a la denominada perspectiva de género, que centra su análisis en las relaciones de género en la actividad laboral y en la escasa participación de las mujeres en el proceso de toma de decisiones y posiciones estratégicas (Fernández et al., 2010).

Bajo estos lineamientos, Powell y Eddleston (2011), sugieren que los hombres y las mujeres gestionen los negocios de forma distinta, por tanto, el sector, el tamaño, la edad organizacional, el carácter familiar, la junta directiva y el número de socios son herramientas de caracterización que condicen a marcar diferencias de gestión. A continuación, se muestran estudios empíricos anteriores relacionados con el ser hombre 0 mujer gerente y las variables que se buscan analizar en esta investigación.

\subsection{La gerencia sea hombre o mujer y el sector}

Dwyer, Richard y Chadwick (2003), plantean que el impacto de la participación de la gerencia sea hombre o mujer depende del contexto organizativo de la empresa. Por tanto, el comportamiento de la PyME según la gerencias sea hombre o mujer da muestra de los sectores donde más actúan cada uno de estos. En los estudios realizados por Fernández et al. (2010), Brenes y Bermúdez (2013) y el The Global Gender Gap Report

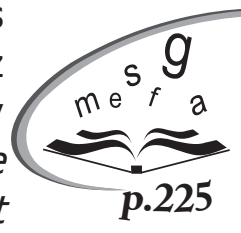

2013 se reconoce que las mujeres directivas desarrollan su actividad de gestión en mayor proporción en los sectores de servicios e industria, y en construcción como último sector; por su parte, los hombres desarrollan 
las mismas actividades directivas, en el mismo orden pero en mayor proporción. Por otro lado, Al Marzouqi y Forster (2011) y Moore, Griffiths, Richardson y Adam (2008), señalan que las mujeres no tienen una buena representación en el sector de la tecnología de la información, porque no han tenido acceso a la educación en carreras que estén orientadas a áreas relacionadas con las tecnologías, lo cual indica que la participación en esta actividad tan específica es limitada.

\subsection{La gerencia sea hombre o mujer y el tamaño de la organización}

Cliff (1998), en sus investigaciones, identifica que las empresas de menor tamaño son dirigidas por mujeres. Otros resultados como los de Danes, Stafford y Loy (2007) y Díaz y Jiménez (2010), señalan también estas diferencias, pero relacionadas con el volumen de ventas y el número de empleados. Además, Orser et al. (2010) identifican que las organizaciones dirigidas por mujeres son de menor tamaño y tienden en mínima proporción al crecimiento, lo cual indica una constante en la relación de las mujeres con el tamaño de la organización. De la misma manera, la investigación realizada por Marinova, Plantenga y Remery (2010) muestra que el número de altos directivos femeninos puede influir positivamente en el desarrollo profesional de las mujeres en puestos inferiores, además de generar mejores beneficios y garantías.

\subsection{La gerencia sea hombre o mujer y la edad de la organización}

Las investigaciones de ser hombre o mujer, y edad de la organización son escasas; sin embargo, Díaz y Jiménez (2010) identifican que las empresas dirigidas por mujeres afrontan ciertas desventajas estructurales, tales como ser empresas con una menor dimensión y antigüedad, y en estos términos genera un menor desarrollo del aprendizaje sobre el comportamiento del mercado.

\subsection{La gerencia sea hombre o mujer y el carácter familiar}

Los estudios realizados por Haddock y Zanna (1994) plantean que muchos directivos son reacios a dar en sucesión la empresa a mujeres, y este es un aspecto que no debería suceder en las empresas familiares. Este tipo de empresas prefieren los estereotipos sociales asociados a la masculinidad para dar una mejor evaluación al gerente hombre. Este estereotipo genera que en el momento de dar el direccionamiento de la empresa familiar se prefiera a un hijo hombre. Por otro lado, las investigaciones recientes como las de Powell y Eddleston (2011) señalan el beneficio sinérgico de las mujeres en el trabajo y la familia al permitirle tener mayores beneficios dentro de la organización. Así mismo, Knörr (2011) reconoce que se han incrementado los negocios donde la propietaria directora es mujer, y señala como principal causa la facilidad con que las mujeres pueden acceder a niveles más altos de dirección y hacer carrera profesional. 


\subsection{La gerencia sea hombre o mujer y la junta directiva}

En los estudios realizados por Marinova, Plantenga y Remery (2010), Nygaard (2011), Hoogendoorn, Oosterbeek y Van Praag (2013) y Ahern y Dittmar (2012), se identifica que la participación de las muje-res que pertenecen a la junta directiva es de $5.4 \%$ en términos más bajos, y de $40 \%$ como máximo. Por otro lado, Dwyer, Richard y Chadwick (2003) y Marinova, Plantenga y Remery (2010), indagan sobre la participación de las mujeres y los hombres en la junta directiva e identifican que esta ofrece ventajas relacionadas con la incorporación de nuevos conocimientos, experiencia, flexibilidad, productividad y rentabilidad para suplir las necesidades del mercado o suplir nuevos segmentos.

\subsection{La gerencia sea hombre o mujer y los socios o dueños}

Las investigaciones de socios de ambos géneros en la propiedad de la organización han llevado a la conclusión de que las mujeres

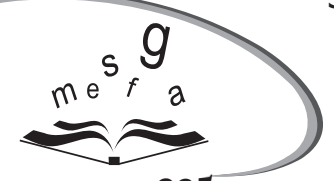

p. 225 suelen mostrar una mayor conciencia ética en la gestión de su empresa (Welch, Welch y Hewerdine, 2008). Además, demostraron que las mujeres emprendedoras tienen un mayor grado de prevención del riesgo que los hombres. Sin embargo, Dwyer, Richard y Chadwick (2003), y Adams y Ferreira (2004), encuentran que la proporción de mujeres en la propiedad de las empresas tiene una influencia positiva en el valor de la empresa; así mismo la participación de las mujeres como accionistas contribuye a alcanzar el éxito de la organización. Según los estudios de Brenes y Bermúdez (2013), los porcentajes de mujeres propietarias de PyMEs son bajos.

\subsection{La gerencia sea hombre o mujer y el nivel de formación}

En términos generales, los estudios de Callejo (2005) indican que las mujeres han encaminado su formación hacia ocupaciones tradicionales, como la enseñanza o la administración; en cambio, el campo de las ciencias aplicadas ha sido considerado casi exclusivo de hombres (Watson, 2002). Por tanto, Dambrin y Lambert (2008) y Adecco (2009), consideran que esta elección se produce por la necesidad de conciliar vida laboral y familiar, donde las mujeres deben elegir profesiones que no impliquen tanta inversión de capital humano ni tantos niveles de formación. Sin embargo, según Brenes y Bermúdez (2013), la distribución porcentual por nivel educativo en general entre mujeres y hombres es similar. El porcentaje más alto en ambos casos corresponde a la formación universitaria, y el más bajo al posgrado. 


\section{Metodología}

D ara esta investigación, se realiza metodología de tipo cuantitativa que permite la utilización de datos de manera objetiva. Para ello, se selecciona una muestra estadística y se procesan los datos que brindan fiabilidad y validez en los resultados. Es tipo de metodología es seleccionado, ya que brinda una aproximación a los resultados más objetiva con relación a las características de las PyMEs y la gerencia.

\subsection{Muestra}

Para la realización de la investigación, se utilizó la base de datos de la Cámara de Comercio de Santiago de Cali. La técnica de recolección de información utilizada fue la encuesta personal y se empleó como soporte un cuestionario autoadministrado dirigido al gerente de la empresa. La población escogida fueron las PyMEs de Santiago de Cali. Según la Cámara de Comercio de Cali, a noviembre de 2011, existen 7376 PyMEs, de las cuales el $80 \%$ son empresas pequeñas y el $20 \%$ son empresas medianas. Para la aplicación de esta investigación, se elige el sector manufacturero, categorizado como sector $\mathrm{C}$, y el sector de alta tecnología, categorizado como sector J, para especificar más el comportamiento de las PyMEs en este tipo de actividad económica. Para estos sectores, en Santiago de Cali, existen 242 PyMEs.

El diseño general de la muestra se fundamenta en los principios del muestreo estratificado en poblaciones finitas. Por ello, el número de criterios y estratos debe ser moderado y congruente con el máximo tamaño de muestra con el que se pueda trabajar. Se emplea el método de muestreo aleatorio estratificado con un nivel de confianza de $93 \%$, un margen de error de $7 \%$ y una proporción de $50 \%$, de la cual se obtuvo que el tamaño adecuado de la muestra para las PyMEs de Santiago de Cali del sector manufactura y de alta tecnología es de 142 empresas.

\subsection{Variables de caracteri- zación}

Para la realización de la investigación, se utilizó como variable independiente el género de la gerencia, la cual fue cruzada con las variables de caracterización de sector, tamaño, edad organizacional, nivel de formación, carácter familiar, junta directiva, y socios o dueños. Cada una de estas variables es descrita a continuación:

- Género de la gerencia: para términos del estudio significa ser hombre o mujer.

- Sector: la Cámara de Comercio de Santiago de Cali reconoce 21 actividades económicas. Para este estudio, se eligió el sector manufacturero y de alta tecnología.

- Tamaño: en esta investigación, el tamaño se mide a partir del número de empleados en 2012 y 2013. Además, dentro del tamaño de la organización, se señala el porcentaje de participación de las mujeres en ella.

- Edad de la organización: esta variable mide el número de años de existencia de la organización desde su fundación. 
- Carácter familiar: para esta investigación, el carácter familiar de la organización se identifica si el grupo familiar posee más de $50 \%$.

- Junta directiva: la junta directiva se evaluó por la existencia de esta dentro de la organización y el número total de miembros.

- Socios o dueños: los socios o dueños son las personas que tienen propiedad sobre la empresa. En este caso, se preguntó por la distribución de mujeres y hombres que hacen parte de la propiedad de la organización.

- Nivel de formación: para esta investigación, la formación del gerente se considera a partir de dos categorías: estudios básicos y estudios universitarios. Dentro de estudios universitarios se especificó la categoría de pregrado, especialización y maestría.

\section{Análisis de resultados y discusión}

\subsection{Análisis descriptivo de las variables caracterizadoras}

Del total de la muestra recogida para el estudio, y tal como sugiere (Tabla 1), las empresas gestionadas por hombres equivalen al $78 \%$, mientras que las gestionadas por las mujeres solo ocupan el $22 \%$. Esto da una participación muy pequeña a la mujer dentro de puestos estratégicos y gerenciales en las PyMEs.

Tabla 1. Empresas según la gerencia sea hombre o mujer

\begin{tabular}{|l|c|c|}
\hline \multicolumn{3}{|c|}{$\begin{array}{c}\text { Empresas según la gerencia sea hombre } \\
\text { o mujer }\end{array}$} \\
\hline & Empresas & $\mathbf{( \% )}$ \\
\hline $\begin{array}{l}\text { Empresas gestionadas } \\
\text { por hombres }\end{array}$ & 111 & 78 \\
\hline $\begin{array}{l}\text { Empresas gestionadas } \\
\text { por mujeres }\end{array}$ & 31 & 22 \\
\hline Total & 142 & 100.0 \\
\hline
\end{tabular}

Fuente. Elaboración propia de los autores.

Por otro lado, (Tabla 2), las variables caracterizadoras muestran que aproximadamente las PyMEs tenían 42 empleados para 2012 y 40 empleados para 2013, lo cual muestra una disminución de empleados no significativa para la gestión organizacional. Así mismo, el porcentaje de participación de los empleados mujeres evidencia un aumento sobre el total de empleados promedio de las empresas. Para 2012, la participación femenina dentro de su fuerza laboral era de $43 \%$, mientras que para 2013 fue de $48 \%$. Estos resultados dan cuenta de que, aunque el número de empleados disminuyó, la participación de la mujer dentro de la fuerza laboral aumentó.

Por otro lado, se da cuenta de que las empresas manufactureras corresponden a $74 \%$, lo cual indica que en Santiago de Cali las PyMEs están en mayor cantidad dedicadas a la industria y aun tienen una mínima participación de organizaciones de alta tecnología. Así mismo, estos resultados demuestran que la edad organizacional es aproximadamente de 20 años, las PyMEs son en 78 $\%$ de carácter familiar y solo $55 \%$ de las empresas tiene junta directiva. Igualmente, las empresas con gerente con formación universitaria corresponden a $89 \%$, de los cuales $58 \%$ solo tiene su título de pregrado. 
Tabla 2. Descriptivos de las variables caracterizadoras

\begin{tabular}{|c|c|c|}
\hline \multicolumn{3}{|c|}{ Descriptivos de las variables caracterizadoras } \\
\hline & 2012 & 2013 \\
\hline Número medio de empleados & 42 & 40 \\
\hline \multirow[t]{2}{*}{$\%$ de empleados mujeres } & $43 \%$ & $48 \%$ \\
\hline & Media & $\begin{array}{c}\text { Desviación } \\
\text { típica }\end{array}$ \\
\hline Miembros de la junta directiva & 6 & 7 \\
\hline \multirow[t]{2}{*}{ Edad de la organización } & 20 & 14 \\
\hline & $\%$ & \\
\hline$\%$ de empresas manufactureras & 74 & \\
\hline \% de empresas de alta tecnología & 26 & \\
\hline$\%$ de empresas de carácter familiar & 78 & \\
\hline$\%$ de empresas con junta directiva & 55 & \\
\hline$\%$ de mujeres socios o dueños & 34 & \\
\hline$\%$ mujeres miembros de la junta directiva & 36 & \\
\hline$\%$ de empresas con gerente con formación universitaria & 89 & \\
\hline \multirow{3}{*}{$\%$ de empresas de acuerdo con la formación universitaria } & Pregrado & 58 \\
\hline & Especialización & 11 \\
\hline & Maestría & 18 \\
\hline
\end{tabular}

Fuente. Elaboración propia de los autores.

El $55 \%$ de las PyMEs posee junta, la cual está conformada por seis personas aproximadamente, de las cuales $36 \%$ son mujeres. Estos resultados coinciden con Marinova, Plantenga y Remery (2010), Nygaard (2011), Hoogendoorn, Oosterbeek y Van Praag (2013) y Ahern y Dittmar (2012), en los que la participación de las mujeres en las juntas directivas oscila entre $5 \%$ y $40 \%$. Del mismo modo, desde el punto de vista de la propiedad, solo el $34 \%$ de los socios o dueños de las PyMEs son mujeres, lo cual puede ser atribuible a las afirmaciones de Welch, Welch y Hewerdine (2008), quienes plantean que las mujeres con deseos de emprender tienen un mayor grado de temor al riesgo que los hombres.
Igualmente, estos resultados son coherentes con el The Global Gender Gap Report 2015, ya que mundialmente la participación femenina dentro de la fuerza laboral no está en condiciones de igualdad frente a los hombres, pero la tendencia es a generar mayores condiciones de participación de hombres y mujeres en el trabajo. En contraste, los resultados de esta investigación señalan que la participación de las mujeres de su propiedad y en junta directiva es limitada, aspecto que coincide con los estudios de Schein, Mueller y Jacobson (1989), y Fernández et al. (2010), quienes sugieren una participación muy baja de las mujeres en cargos estratégicos, aunque el The Global Gender Gap Report 2013 ubique a Colombia en condiciones de igualdad frente a la ocupación de cargos directivos, y esto no sea claro para los resultados de esta investigación. 


\subsection{Análisis descriptivo de las variables caracterizadoras según la gerencia sea hombre o mujer}

La variable de carácter familiar de la empresa indica que $75.7 \%$ de las PyMEs son dirigidas por hombres (Tabla 3). Estas afirmaciones coinciden con los resultados de Haddock y Zanna (1994), y Fernández et al. (2010), en los cuales se reconoce que hay reticencias a dar en sucesión una empresa familiar a una mujer a causa de los estereotipos sociales asociados a la masculinidad. Bajo la línea de empresas gestionadas por hombres o mujeres, los resultados dan muestra de que el carácter familiar de la empresa se presenta principalmente en las que son gestionadas por hombres. Estas afirmaciones no dan cuenta de los resultados dados por Powell y Eddleston (2011) y Knorr (2011), que plantean la tendencia de las mujeres a tener empresas de carácter familiar por su balance vida personal/ laboral. Sin embargo, es necesario atribuir el carácter emprendedor de las mujeres por este tipo de organizaciones pero que no se ven reflejado en los resultados.

Tabla 3. Análisis de las empresas según la gerencia sea hombre o mujer

\begin{tabular}{|c|c|c|}
\hline \multicolumn{3}{|c|}{ Análisis de las PyMEs según la gerencia sea hombre o mujer } \\
\hline & \multicolumn{2}{|c|}{ Empresa gestionada por } \\
\hline & Hombre & Mujer \\
\hline Edad de la organización (promedio de empresas) & 20 & 21 \\
\hline Carácter familiar (\% de empresas familiares) & 75.7 & 24.3 \\
\hline \multicolumn{3}{|l|}{ Sector } \\
\hline Sector manufacturero (\% de empresas) & 78.1 & 21.9 \\
\hline Sector de alta tecnología (\% empresas) & 77.8 & 22.2 \\
\hline \multicolumn{3}{|l|}{ Empleados } \\
\hline Empleados 2012 (promedio de empresas) & 42.0 & 43.0 \\
\hline Empleados 2013 (promedio de empresas) & 39.0 & 48.0 \\
\hline Empleados mujeres en la empresa 2012 (\% de participación) & 42.9 & 51.2 \\
\hline Empleados mujeres en la empresa 2013 (\% de participación) & 46.2 & 45.8 \\
\hline \multicolumn{3}{|l|}{ Junta directiva } \\
\hline Junta directiva (\% de existencia en la empresa) & 79.7 & 20.3 \\
\hline Junta directiva (\% de miembros mujeres) & 34.7 & 40.8 \\
\hline \multicolumn{3}{|l|}{ Socios o dueños } \\
\hline Socios o dueños mujeres (promedio de \% de participación) & 30.6 & 46.0 \\
\hline Socios o dueños hombres (promedio de \% de participación) & 69.4 & 54.0 \\
\hline \multicolumn{3}{|l|}{ Formación del gerente } \\
\hline Formación del gerente (\% de empresas con gerente con estudios básicos) & 68.75 & 31.25 \\
\hline Formación del gerente (\% de empresas con gerente universitario) & 79.4 & 20.6 \\
\hline Formación del gerente en pregrado (\% de empresas) & 66.0 & 61.5 \\
\hline Formación del gerente en especialización (\% de empresas) & 13.0 & 11.5 \\
\hline Formación del gerente en maestría (\% de empresas) & 20.0 & 23.1 \\
\hline
\end{tabular}

Fuente. Elaboración propia de sus autores. 
La edad de la organización no varía significativamente con relación a si la gerencia es hombre o mujer, lo cual no se especifica en investigación previa y no sería una variable caracterizadora de la organización de acuerdo con la gerencia sea hombre o mujer para las PyMEs del sector manufacturero y de alta tecnología de Santiago de Cali.

Los sectores manufacturero y de alta tecnología de las PyMEs de Santiago de Cali son gestionados principalmente por hombres en $78.1 \%$ y $77.8 \%$, respectivamente, mientras que las mujeres tienen niveles más bajos. Por tanto, se puede decir que el sector de alta tecnología, aunque es una actividad económica relativamente nueva, tiene estereotipos masculinos de gestión, lo cual va de la mano con los argumentos de Adams y Ferreira (2004), quienes indican la poca representación de las mujeres en este sector, debido al poco acceso a la formación de carreras asociadas al área. Por su parte, estos resultados contribuyen a los estudios de Brenes y Bermúdez (2013), que evidencian que no hay diferencia significativa entre el sector servicios e industrial en las empresas gestionadas por mujeres.

El número medio de empleados de las empresas gestionadas por hombres son 42 para 2012 y 39 para 2013; sin embargo, el número medio de empleados de las empresas gestionadas por mujeres es de 43 para 2012 y 48 para 2013. En principio, este resultado muestra que las PyMEs de Santiago de Cali gestionadas por hombres son de menor tamaño que las gestionadas por mujeres, aunque no de manera significativa. En contraste, la tendencia de las PyMEs gestionadas por hombres es a reducir el número de empleados por año, mientras que la de las mujeres es a aumentarlo.
La participación de empleados mujeres en las empresas gestionadas por mujeres es mayor con $51.2 \%$ en comparación con las gestionadas por los hombres de $49.2 \%$ para 2012. Para 2013, las empresas gestionadas por hombres aumentaron su participación de empleados mujeres a $46.2 \%$. Un caso contrario sucede con la participación de los empleados mujeres en las empresas gestionadas por mujeres, que disminuyó a $45.8 \%$. En decir, las mujeres tienen más empleados y tienden a incrementar la participación masculina, y los hombres tienen menos empleados pero tienden a incrementar la participación femenina.

La variable de la junta directiva muestra que la existencia de esta en las PyMEs es de $79.7 \%$ en empresas gestionadas por hombres. En cambio, la participación de las mujeres en las juntas directivas tiene una mayor representación cuando son empresas gestionadas por mujeres, con $40.8 \%$. Estos resultados se ajustan a los resultados de Knorr (2011), donde plantea que, cuando las mujeres ocupan cargos de alta dirección, suelen aumentar el número de participación femenina en cargos directivos para incrementar los beneficios hacia esta.

Los socios o dueños mujeres de las PyMEs de Santiago de Cali suelen ser mayoría cuando las empresas son gestionadas por mujeres, en comparación con las gestionadas por los hombres con $46 \%$ frente a $30.6 \%$, (Tabla 3 ). Por otra parte, las empresas gestionadas por mujeres tienen mayor participación masculina que femenina. En cuanto a las empresas gestionadas por hombres, la representación masculina de los socios es de $69.4 \%$, lo cual indica que tanto las empresas gestionadas por hombres como las gestionadas por mujeres tienen una participación de socios en su mayoría masculina. 
En este sentido, la diferencia cuando una empresa es gestionada por hombres es más significativa con relación a la participación de mujeres socias o dueñas gracias a sus preferencias por los hombres. Estos resultados coinciden con los de Welch, Welch y Hewerdine (2008), que señalan que las mujeres suelen tener más temor al emprendimiento $y$, por tanto, su participación en la empresa es mínima.

El nivel de formación del gerente sugiere que en las empresas gestionadas por hombres, en $79.4 \%$ su nivel de formación es universitario. En contraste, $20.6 \%$ de los gerentes con formación universitaria gestionan PyMEs. Por tanto, las empresas gestionadas por mujeres tienen más gerentes en estudios básicos que universitarios. Estos resultados se asocian directamente con los datos del Progrma de la Naciones Unidas para el Desarrollo (PNUD, 2013), que indica que la actividad económica de las mujeres es más baja que la de los hombres en Santiago de Cali y su nivel de formación no es tan alto.

Los resultados anteriores pueden ser atribuidos a las afirmaciines dadas por Dambrin y Lambert (2008) y Adecco (2009), que plantean que las mujeres suelen inducir a menos niveles de formación a causa de su necesidad de conciliar la vida laboral con la vida familiar y a sus ocupaciones asignadas tradicionalmente. Del mismo modo, los resultados de esta investigación muestran que los niveles de formación de pregrado, especialización y maestría no varían significativamente cuando las empresas son gestionadas por hombres o mujeres. Por otro lado, este resultado se contrasta con los datos de Villamizar García-Herreros (2011), los cuales sugieren que en Colombia los hombres tienen más nivel de formación profesional que las mujeres. También plantea esta diferencia profesional dada por la incursión de la mujer en el mercado laboral, la cual se da por la exigencia por un nivel de formación profesional igual a la de los hombres pero con una brecha salarial aún amplia. En este sentido, se aborda que las diferencias de formación profesional están dadas por la ubicación en zona rural o urbana según muestra el estudio de esta misma institución.

Es evidente que los porcentajes donde ambas partes tienen niveles elevados son en la formación de pregrado, resultado coherente con Brenes y Bermúdez (2013), quienes indican que la formación universitaria para mujeres y hombres es similar y tiene mayor proporción en formación de pregrado que en posgrado. 


\section{Conclusiones}

- sta investigación da cuenta de la inciCdencia del gerente hombre o mujer en las características de las PyMEs de los sectores de manufactura y alta tecnología de Santiago de Cali. Los resultados exponen el comportamiento de las PyMEs según las variables de caracterización que hasta ahora se tienen de un estudio más grande. Se evidencia que el comportamiento por el sector, el tamaño, la edad de la organización, el carácter familiar, la junta directiva, los socios y los dueños, y el nivel de formación tiene relación con que la gerencia sea hombre o mujer.

Los resultados muestran que las empresas que son dirigidas por mujeres no tienen gran peso en el sector manufacturero ni de alta tecnología $-21.9 \%$ y $22.2 \%$, respectivamente-, un mayor tamaño con relación al número de empleados -48 empleados-, tienen una menor participación femenina en la fuerza laboral a lo largo del tiempo -de 51.2 $\%$ a $45.8 \%$ entre 2012 y $2013-$, no suelen tener junta directiva -solo $20.3 \%$ - y si la tienen la participación de las mujeres en ella es mayor a la de los hombres $-40.8 \%-$, son pocas de carácter familiar $-24.3 \%-$, tienen más socios o dueños hombres $-54 \%$-, y el nivel de formación del gerente es en mayor proporción a estudios básicos -31.2 \%y, además, cuando poseen estudios universitarios tienen un mayor porcentaje en estudios de pregrado -61.5 \%-.

Una de las principales conclusiones que estos resultados evidencian es que el papel laboral de las mujeres sigue marcado por la orientación a ser empleada y no a emplear. Es decir, que desde el punto de vista de la distribución del poder y la autoridad, las mujeres tienden a mantener posiciones de obediencia y no de mando, tal como lo mencionan las investigaciones de Eagly (1987).

Las evidencias sugieren que las mujeres tienen posibilidades menores de pertenecer a una empresa familiar, de tener un nivel de formación y de pertenecer a todo tipo de actividad económica, a pesar de que las reglamentaciones colombianas favorezcan el carácter emprendedor. Muchas de estas atribuciones son dadas desde la metáfora del techo de cristal (Barnet-Verzar y Wolf, 2008), que expone el no acceso de las mujeres a todo este tipo de oportunidades.

Es interesante también que los resultados muestren que las mismas mujeres tengan un estereotipo de acceso y éxito al mundo empresarial ligado a la masculinidad. Las mujeres gerentes prefieren, dentro de su grupo estratégico, a otras mujeres, pero no dentro de actividades tácticas. Lo anterior puede posibilitar investigaciones que en el futuro examinen las relaciones de diversidad en los grupos y la forma de relación entre empleadores y empleados del mismo género, y la forma en cómo las personas del mismo género dentro de la organización se ayudan o generan obstáculos.

Esta investigación busca contribuir al desarrollo de estudios de género en la PyME de Santiago de Cali y dar un resultado más específico para la aplicación de políticas de igualdad en ciertos sectores. No se puede dejar de lado que este tipo de investigaciones permite aplicar a otras actividades económicas que podrían brindar resultados similares. Igualmente, este estudio no está concluido, sino que es la primera parte de un proceso de investigación que se orientará a identificar 
cómo el ser hombre o mujer gerente incide en las diferentes características de las organizaciones, con el objeto de desarrollar estudios empíricos que permitan aportar al desarrollo teórico y práctico del estudio.

Los resultados de esta investigación pueden ser utilizados para adoptar medidas de administración del personal y del manejo de la cultura organizacional. Contribuye a la selección de personal y gerentes por ser hombre o mujer según las estrategias de gestión que se busquen en las organizaciones. Sin embargo, tiene limitaciones que pueden considerarse para futuros trabajos. Las proximas investigaciones también pueden establecer temas de diversidad de género para establecer conexiones entre problemas de igualdad y equidad. En principio, la información suministrada está directamente asociada con información específica de las PyMEs de Santiago de Cali y al tipo de sector elegido. Además, dentro de las empresas seleccionadas, la muestra de mujeres que gestionan la empresa son en menor proporción que la de los hom-bres, lo cual influye directamente en los resultados frente al género, pero brinda una caracterización de las organizaciones gestionadas por hombres o mujeres. 


\section{Referencias}

Adams, R. B. y Ferreira, D. (2004). Gender diversity in the boardroom. ECGI Working Paper Series in Finance, 57. Recuperado de http://www.cfr-cologne.de/download/ researchseminar/SS2006/genderECGI.pdf

Adecco (2009). III Encuesta Adecco a Mujeres Directivas. Recuperado de http://www. adecco.es/_data/estudios/pdf/485.pdf

Ahern, K. R. y Dittmar, A. K. (2012). The changing of the boards: The impact on firm valuation of mandated female board representation. The Quarterly Journal of Economics, 127(1), 137-197.

Al Marzouqi, A. H. y Forster, N. (2011). An exploratory study of the under-representation of Emirate women in the United Arab Emirates' information technology sector. Equality, Diversity and Inclusion: An International Journal, 30(7), 544-562.

Barnet-Verzat, C. y Wolff, F. C. (2008). Gender wage gap and the glass ceiling effect: A firm-level investigation. International Journal of Manpower, 29(6), 486-502.

Brenes Bonilla, L. y Bermúdez Mesén, L. (2013). Diferencias por género en el emprendimiento empresarial costarricense. TEC Empresarial, 7(2), 19-27.

Callejo, J. (2005). Disponibilidad temporal corporativa y género: aproximaciones empíricas. Anduli, 4, 31-59.

Campbell, K. y Minguez-Vera, A. (2007). The influence of gender on spanish boards of directors: An empirical analysis. IVIE Working paper, WP-EC 2007-08. IVIE.
Castaño, C., Martín, J., Vázquez, S. y Martínez, J. L. (2010). Female executives and the Glass Ceiling in Spain. International Labour Review, 149(3), 343-360.

Cliff, J. E. (1998). Does one size fit all? Exploring the relationship between attitudes towards growth, gender, and business size. Journal Of Business Venturing, 13(6), 523-542.

Congreso de Colombia. Ley 731 de 2002. Por la cual se dictan normas para favorecer a las mujeres rurales (16 enero 2002).

Congreso de Colombia. Ley 823 de 2013. Por la cual se dictan normas sobre igualdad de oportunidades para las mujeres (7 octubre 2003).

Cuadrado, I. y Francisco Morales, J. (2007). Algunas claves sobre el techo de cristal en las organizaciones. Revista de Psicología del Trabajo y de las Organizaciones, 23(2), 183-202.

Dambrin, C. y Lambert, C. (2008). Mothering or auditing? The case of two big Four in France. Accounting, Auditing \& Accountability Journal, 21(4), 474-506.

Danes, S. M., Stafford, K. y Loy, J. T. C. (2007). Family business performance: The effects of gender and management. Journal of Business Research, 60(10), 1058-1069.

Department of Economic and Social Affairs (2010). The World's Women 2010: Trends and Statistics. Nueva York: United Nations.

Díaz García, M. C. y Jiménez Moreno, J. J. (2010). Recursos y resultados de las peque- 
ñas empresas: nuevas perspectivas del efecto género. Cuadernos de Economía y Dirección de la Empresa, 13(42), 151-175.

Driga, O. y Prior, D. (2010). Start-Up conditions and the performance of women-and mencontrolled businesses in manufacturing industries. Revista de Contabilidad, 13(1), 89-123.

Du Rietz, A. y Henrekson, M. (2000). Testing the female underperformance hypothesis. Small Business Economics, 14(1), 1-10.

Dwyer, S., Richard, O. C. y Chadwick, K. (2003). Gender diversity in management and firm performance: The influence of growth orientation and organizational culture. Journal of Business Research, 56(12), 1009-1019.

Eagly, A. H. (1987). Sex differences in social behavior: $A$ social-role interpretation. Hillsdale, NJ: Erlbaum.

Fernández Palacín, F., López Fernández, M., Maeztu Herrera, I., Martín Prius, A. (2010). El techo de cristal en las pymes. Revista de Estudios Empresariales, 1, 231-247.

Fuentes García, F. J. y Sánchez Cañizares, S. M. (2010). Análisis del perfil emprendedor: una perspectiva de género. Estudios de Economía Aplicada, 28(3), 1-28.

Haddock, G. y Zanna, M. P. (1994). Preferring "housewives" to "feminists": Categorization and the favorability of attitudes toward women. Psychology of Women Quarterly, 18(1), 25-52.

Hoogendoorn, S., Oosterbeek, H. y Van Praag, M. (2013). The impact of gender diversity on the performance of business teams: Evidence from a field experiment. Management Science, 59(7), 1514-1528.

Jimeno, F. J. y Redondo, M. (2007). Diversidad de género en el consejo de administración y características económico-financieras de las empresas. Ponencia presentada en XIV Congreso de la Asociación Española de Contabilidad y Administración de Empresas, Valencia, España.

Justo, R. (2007). La influencia del género en el fracaso empresarial: una aplicación al caso de España. Revista de Empresa: La fuente de ideas del ejecutivo, 20, 52-64.

Knörr, H. (2011). From top management to entrepreneurship: Women's next move? International Journal of Manpower, 32(1), 99-116.

Lamas, M. (1994). Cuerpo: diferencia sexual y género. Debate Feminista, 10, 3-31.

Marinova, J. H., Plantenga, J. y Remery, C. L. H. S. (2010). Gender diversity and firm performance: Evidence from dutch and danish boardrooms. Discussion Paper Series, 10(3).

Moore, K., Griffiths, M., Richardson, H. y Adam, A. (2008). Gendered futures? Women, the ICT workplace and stories of the future. Gender, Work \& Organization, 15(5), 523542.

Moser, C. O. (1989). Gender planning in the Third World: Meeting practical and strategic gender needs. World Development, 17(11), 1799-1825.

Nygaard, K. (2011). Forced board changes: Evidence from Norway. Recuperado de https://papers.ssrn.com/sol3/papers. cfm?abstract_id=1793227 
Orser, B; Spence, M; Riding, A \& Carrington, C. (2010). Gender and export propensity. Entrepreneurship Theory and Practice, 34(5), 933-957.

(PNUD) Programa de la Naciones Unidas para el Desarrollo (2013). iIgualdad Salarial entre hombres y mujeres en Santiago de Cali? De la legislación a la realidad. Bogotá: PNUD.

Powell, G. N. y Eddleston, K. A. (2011). Work family enrichment and entrepreneurial success: Do female entrepreneurs benefit most? The Academy of Management, 32(3), 747-760.

Rose, C. (2007). Does female board representation influence firm performance? The Danish evidence. Corporate Governance: An International Review, 15(2), 404-413.

Schein, V., Mueller, R. y Jacobson, C. (1989). The relationship between sex role stereotypes and requisite management characteristics among college students. Sex Roles, 20, 103-110.

Torres González, O. y Pau, B. (2012). "Techo de cristal" y "suelo pegajoso": la situación de la mujer en los sistemas alemán y español de ciencia y tecnología. Revista Iberoamericana de Ciencia Tecnología y Sociedad, 6(18), 35-59.

Villamizar García-Herreros, M. U. (2011). Uso del tiempo de mujeres y hombres en Colombia: midiendo la inequidad. Santiago de Chile: Naciones Unidas. Recuperado de http://www.cepal.org/publicaciones/ xml/3/42803/Serie107.pdf

Watson, J. (2002). Comparing the performance of male-and female-controlled businesses: Relating outputs to inputs. Entrepreneurship: Theory and Practice, 26(3), 91-101.
Welch, C. L., Welch, D. E. y Hewerdine, L. (2008). Gender and export behaviour: Evidence from women-owned enterprises. Journal of Business Ethics, 83(1), 113-126.

Woodhams, C. y Lupton, B. (2009). Analysing gender-based diversity in SMEs. Scandinavian Journal of Management, 25(2), 203-213.

World Economic Forum (2013). The Global Gender Gap Report 2013. Recuperado de https://www.weforum.org/reports/globalgender-gap-report-2013

Perdomo C., G., Rosas Castro, J. A. y Murillo Vargas, G. (2014). Análisis del cambio organizacional e institucional: caso CREAME (1996-2010), en Medellín, Colombia. Cuadernos de Administración, 30(51), 25-35. Recuperado de http:// www.scielo.org.co/scielo.php?script=sci abstract\&pid $=$ S012046452014000100004

Pinto Pinto, L. (2012). Estado del arte sobre la creación y gestión de spin-off universitarias. Bucaramanga, Colombia: Universidad Industrial de Santander. Recuperado de https://slidedoc.es/estado-delarte-sobre-la-creacion-y-gestion-de-spinoff-universitarias-pdf

Project Management Institute, Inc. (2013). PmBok. Guía de los fundamentos para la dirección de proyectos $P M B O K^{\circledR}$ (4. ${ }^{\mathrm{a}}$ ed.). Newtown Square, Pensilvania: Project Management Institute.

Universidad del Valle (2012). Modelo spinoff en biotecnología en la Universidad del Valle. Recuperado de http://www. altec2013.org/programme_pdf/371.pdf 